\title{
Entangled and sequential quantum protocols with dephasing
}

\author{
Sergio Boixo ${ }^{1}$ and Chris Heunen ${ }^{2}$ \\ ${ }^{1}$ Harvard University and University of Southern California \\ ${ }^{2}$ Oxford University
}

\begin{abstract}
Sequences of commuting quantum operators can be parallelized using entanglement. This transformation is behind some optimal quantum metrology protocols and recent results on quantum circuit complexity. We show that dephasing quantum maps in arbitrary dimension can also be parallelized. This implies that for general dephasing noise the protocol with entanglement is not more fragile than the corresponding sequential protocol and, conversely, the sequential protocol is not less effective than the entangled one. We derive this result using tensor networks. Furthermore, we only use transformations strictly valid within string diagrams in dagger compact closed categories. Therefore, they apply verbatim to other theories, such as geometric quantization and topological quantum field theory. This clarifies and characterizes to some extent the role of entanglement in general quantum theories.
\end{abstract}

One of the main goals of quantum computation and quantum information is the understanding of entanglement and its use to surpass the classical bounds for some given task. Quantum metrology is a case in point. It tries to exploit entanglement to measure physical parameters of a system to high precision [1 60]. Consider, for example, the phase estimation problem in which one is given a phase gate $e^{-i \phi \sigma_{z} / 2}$ as a black box and one is to find the corresponding phase $\phi$. A standard method to approach this problem is the so-called Ramsey interferometry, where the unitary $e^{-i \phi \sigma_{z} / 2}$ is applied to each subsystem of an initial quantum state $(|0\rangle+|1\rangle)^{\otimes n} / \sqrt{2^{n}}$. We are interested in the scaling with $n$ of the uncertainty $\delta \hat{\phi}$ of the estimator $\hat{\phi}$. In Ramsey interferometry $\delta \hat{\phi} \propto 1 / \sqrt{n}$, a scaling called the shot noise or standard quantum limit. In fact, as long as the initial state remains separable and one operator is applied to each subsystem, this bound cannot be surpassed (see supplementary material).

One way to improve this scaling is to introduce entanglement on the probe. The initial state, prior to the evolution, is now transformed into $(|0 \cdots 0\rangle+|1 \cdots 1\rangle) / \sqrt{2}$. This state can be achieved with an entangling operator (as described in detail below). Applying the phase gate to each subsystem and then a disentangling operator we obtain $\left(|0\rangle+e^{-i n \phi}|1\rangle\right) \otimes|0 \cdots 0\rangle / \sqrt{2}$. The uncertainty scaling is now $\delta \hat{\phi} \propto 1 / n$, which is called the Heisenberg limit 26, 31]. Intuitively, entanglement in the probe improves the measurement sensitivity [2]. This principle has been corroborated experimentally on several occasions [14, 15, 35].

The scaling $\delta \hat{\phi} \propto 1 / n$ can also be obtained in a different way. The final state of the first qubit in the entangled protocol is $\left(|0\rangle+e^{-i n \phi}|1\rangle\right) / \sqrt{2}$. The same state results when acting with $n$ sequential phase gates $\left(e^{-i \phi \sigma_{z} / 2}\right)^{n}=e^{-i n \phi \sigma_{z} / 2}$ on the first qubit initialized to $(|0\rangle+|1\rangle) / \sqrt{2}$. Notice that, if with a single application of the phase gate per measurement the uncertainty is constant $\delta \hat{\phi} \propto 1$, then with $n$ sequential unitaries the uncertainty is $\delta(n \hat{\phi}) \propto 1$, giving $\delta \hat{\phi} \propto 1 / n$. This sequential version has been explored in frame synchronization [13] and clock synchronization [23, 29] between two parties.

Given a unitary operator $U$ as a black box and an input state $|\psi\rangle=\sum_{j} c_{j}\left|e_{j}\right\rangle$, where $\left|e_{j}\right\rangle$ are eigenvectors of $U=\sum e^{i \varphi_{j}}\left|e_{j}\right\rangle\left\langle e_{j}\right|$, Kitaev's phase estimation algorithm [61] outputs an approximation of the phase $\varphi_{j}$ corresponding to $\left|e_{j}\right\rangle$ with probability $\left|c_{j}\right|^{2}$. This algorithm is known to be optimal [33], and it can also be transformed into the sequential protocol [62]. The sequentialization is done by changing the quantum Fourier transform used in Kitaev's phase estimation into a semiclassical Fourier transform [63].

The transformation between sequential and entangled protocols has also been used to study the class QNC of quantum circuits with polylogarithmic depth 64]. This class includes, for instance, standard quantum errorcorrection encoding and decoding. Interestingly, if we had entangling gates with arbitrary fan-out at our disposal, then certain important functions that require classical circuits of logarithmic depth could be computed by quantum circuits of constant depth [65].

In this paper we study the relation between entangled and sequential protocols using tensor networks 6672], which encompass similar notations for Liouville space [3], quantum games [74] and the so-called quantum combs 75,76$]$. We first introduce this notation for protocols with unitary operators. The diagrams derived are indeed very general, and they hold verbatim for quantum maps. Therefore, we extend the relation between entangled and sequential protocols to the presence of noise.

One reason for the generality of the diagrams used is that we only employ transformations strictly valid within string diagrams in dagger compact closed categories. Consequently, they apply to other quantum theories where the maps are not tensors (for instance, they can be of topological nature). While we refer to the literature for a formal introduction to this subject [77 81], for the purpose of this paper it suffices to say that dagger compact closed categories capture exactly the necessary mathematically structure that makes the manipulations with string diagrams possible. In particular, composition is denoted by connecting maps, there is an abstract ten- 
sor structure between maps indicated by drawing maps in parallel, an abstract dagger action is represented by switching the input and the output of a map, and lines (the input/output of operations) can cross. All these operations are compatible between them, so the intuition gained with tensor diagrams still applies. Reciprocally, for tensor networks of quantum maps we will also use some adornments introduced in the context of completely positive categories which keep track (and partly explain) the emergence of transpose operations when carrying out certain manipulations (see Eq. 9).

For a given Hilbert space $\mathcal{H}$ and a choice of basis $\{j\}$ the isometry $\delta=\sum_{j}|j j\rangle\langle j|$ is an entangling operator. We denote it by -2 . This is a generalization of the following quantum circuit:

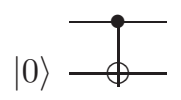

We now characterize commuting operators in finitedimensional Hilbert spaces. States are represented by the diagram $\bigcirc$ - For a given state $|a\rangle$, we can use the entangling operator to obtain a new operator

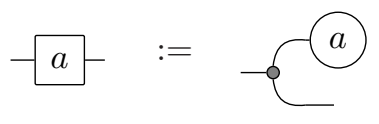

which explicitly acts as

$$
\sum_{j}|x\rangle \mapsto \sum_{j}\langle a \mid j\rangle\langle j \mid x\rangle|j\rangle=\left\langle a\left|\left(\sum_{j}|j j\rangle\langle j|\right)\right| x\right\rangle .
$$

Commuting operators correspond exactly to different states with the same entangling operator or choice of basis. These operators also commute with the entangling operator (see supplementary material for a diagrammatic proof based on the associative property of $\delta$.)

$$
\begin{aligned}
a-\{ & =\left\{\begin{array}{l}
a \\
\sum_{j}|j j\rangle\langle j|a| j\rangle\langle j|
\end{array}=\sum_{j}(a \otimes 1)|j j\rangle\langle j|\right.
\end{aligned}
$$

The physical interpretation is that there is no difference whatsoever between applying a commuting gate first and then the entangling operator or first entangling and then applying the gate.

Writing o- for the (unnormalized) equal superposition state $\sum|j\rangle$ for the given choice of basis, and $\rightarrow$ for its adjoint $\sum\langle j|$, we obtain equivalence between the following two diagrams for any set of commuting operators $\left\{f_{j}\right\}$ :

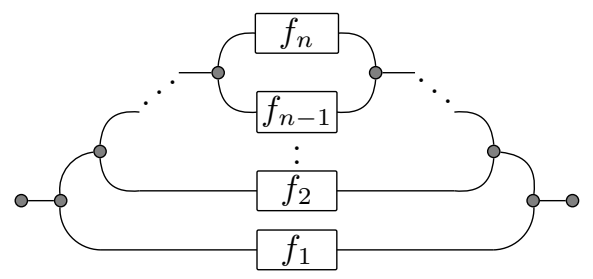

$$
f_{\pi(1)} f_{\pi(2)}-\cdots-f_{\pi(n-1)} f_{\pi(n)} \circ
$$

for any permutation $\pi \in S(n)$. Diagram (5) corresponds to the entangled protocol in three steps: entangling gate, commuting gate acting on each component, disentangling gate. Diagram (6) clearly corresponds to the sequential protocol. The equivalence follows diagrammatically because every commuting operator $f_{j}$ can be moved to the beginning of the diagram, and the remaining loops can be cancelled by the isometry property $-0-=$ (See supplementary material). Explicitly, the main step is as follows:

$$
\sum_{k, l}|k\rangle\left\langle k k\left|1 \otimes f_{j}\right| l l\right\rangle\langle l|=f_{j}=\left(\sum_{k, l}|k\rangle\langle k k \mid l l\rangle\langle l|\right) \circ f_{j},
$$

where $f_{j}$ can be represented as in Eq. 2. Note that this equivalence also establishes the commutativity of the operators among themselves.

We have shown that the operators defined by the diagram of Eq. (2) commute with the entangling gate. Using the (unnormalized) equal superposition state, we can prove the converse. Given an operator $a$ that commutes with an entangling operator, it is easy to see that the following diagram defines the corresponding state (see supplementary material):

$$
\text { a }-:=a
$$

Explicitly, the state $|a\rangle=\sum_{j} a|j\rangle$ and the operator $a$ are related by Eq. (2).

We have given a complete diagrammatic characterization of all the commuting operators and reviewed the known equivalence of the corresponding entangled and sequential protocols. We now extend this equivalence to quantum maps with general dephasing (a similar question has been addressed before for maps on qubits [23]). To distinguish $\mathcal{H}$ from its dual space $\mathcal{H}^{*}$, we annotate the corresponding wires in diagrams with opposite arrows. Thus operators $c$ from $\mathcal{H}_{A}$ to $\mathcal{H}_{B}$ come in four variations: the original, the transpose $c^{*}$, the adjoint $c^{\dagger}$, and the conjugate $c_{*}=\left(c^{\dagger}\right)^{*}$.

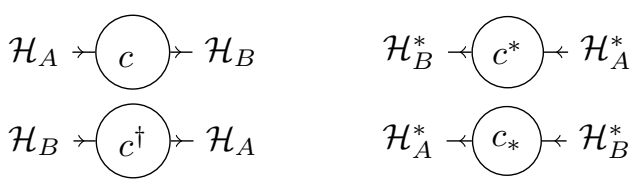

We can now use the notation $($ for the (entangled) state $\sum_{j}|\tilde{j} j\rangle \in \mathcal{H}^{*} \otimes \mathcal{H}$, and $\mathcal{j}$ for its adjoint.

Now, let $\mathcal{A}(\rho)=\sum_{t} a_{t} \cdot \rho \cdot a_{t}^{\dagger}$ be a completely positive map with Kraus operators $\left\{a_{t}\right\}$. Define the operator $a=$ $\sum_{t}|t\rangle \otimes a_{t}$ (where the states $|t\rangle$ live in a environment Hilbert space $\mathcal{H}_{E}$ ). Its diagrammatic representation is

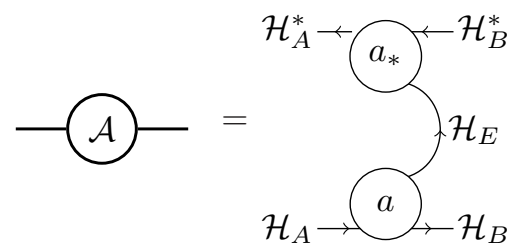


The second diagram corresponds to the Stinespring representation of $\mathcal{A}$. All completely positive operators can be put in this form. The thick first diagram is defined as a more concise representation. Positive states $B=b^{\dagger} b$ also have this form, with trivial input space $\mathcal{H}_{A}=\mathbb{C}$.

Every operator between Hilbert spaces can be lifted to a completely positive quantum map. The quantum map obtained by lifting the entangling operator $\delta$ for a given choice of basis has the form

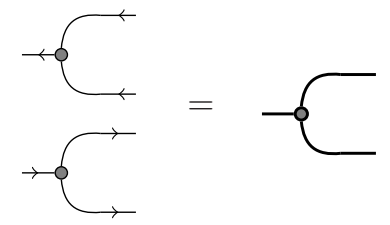

For any positive state and a choice of basis we can then characterize all commuting quantum maps in that basis. They are obtained exactly as in the diagrams of Eq. (2), and take the form

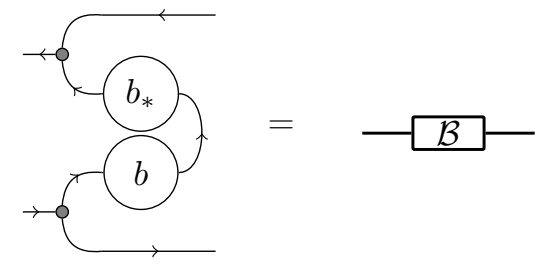

Notice that the diagrammatic proofs (as in the supplementary material) stay exactly the same, whereas the direct computations as in Eq. (7) become rather more involved.

All the diagrams introduced for unitary operators that commute with the entangling operator apply verbatim to quantum maps. Indeed, the quantum maps constructed through an entangling map commute and all maps commuting with an entangling map are of this particular form. Further, the equivalence between the entangled and sequential protocols is upheld.

We now give explicit equations for quantum maps that commute with an entangling map. From the defining diagram Eq. (12) we see that the quantum map $\mathcal{B}$ corresponding to a positive operator $B$ acts as

$$
\mathcal{B}(\rho)=B^{*} \circ \rho
$$

where $\circ$ is the Schur product in the basis of the entangling map. All quantum maps of that form are completely positive (already shown diagrammatically), but in addition we want them to be trace preserving, which imposes

$$
B_{j j}=1 \text {. }
$$

The positive operator $B$ has a spectral decomposition

$$
B=\sum_{s}\left|\chi_{s}\right\rangle\left\langle\chi_{s}\right|
$$

This gives the Kraus decomposition of $\mathcal{B}(\rho)=\sum_{s} b_{s} \cdot \rho \cdot b_{s}^{\dagger}$, with Kraus operators

$$
b_{s}=\sum_{j}\left\langle\chi_{s} \mid j\right\rangle|j\rangle\langle j|
$$

Notice that in this representation the commuting quantum maps also have commuting Kraus operators.

For unitary phase maps the matrix $B$ must have the form

$$
\left(B_{\text {phases }}\right)_{j k}=e^{-i\left(\phi_{j}-\phi_{k}\right)}|j\rangle\langle k|
$$

for any choice of phases $\left\{\phi_{j}\right\}$. Maps with dephasing with respect to the basis of the entangling map are more interesting. The noise must be dephasing because it does not alter the populations, due to Eq. (13):

$$
(\mathcal{B}(\rho))_{j j}=B_{j j} \rho_{j j}=\rho_{j j} .
$$

For the qubit case (i.e. when the dimension of the Hilbert space is two), the positive matrix $B$ has the form

$$
\left(\begin{array}{cc}
1 & e^{-\gamma-i \phi} \\
e^{-\gamma+i \phi} & 1
\end{array}\right)
$$

where $\phi$ is a phase and $\gamma \geq 0$ parametrizes the dephasing. This quantum channel is

$$
e^{-i \phi \sigma_{z} / 2} \cdot\left((1-p) \rho+p \sigma_{z} \rho \sigma_{z}\right) \cdot e^{-i \phi \sigma_{z} / 2},
$$

where $p=\frac{1-e^{-\gamma}}{2}$ can be interpreted as the probability of a random phase flip.

We can also write explicitly a more generic map that is fully sequentializable. We do that by defining appropriate (unnormalized) vectors $\left|\chi_{s}\right\rangle$ for the positive matrix $B=\sum_{s}\left|\chi_{s}\right\rangle\left\langle\chi_{s}\right|$. These vectors should be orthogonal and for the trace preserving condition should obey

$$
B_{j j}=\sum_{s}\left\langle j \mid \chi_{s}\right\rangle\left\langle\chi_{s} \mid j\right\rangle=\sum_{s}\left|\left(\chi_{s}\right)_{j}\right|^{2}=1
$$

Denote the $n$th roots of unity by

$$
\omega_{j}=e^{-i 2 \pi j / n},
$$

where $n$ is the dimension of the underlying Hilbert space. Define

$$
\left|\chi_{s}\right\rangle=\sqrt{r_{s}} \sum_{j} e^{-i \phi_{j}} \omega_{j}^{s}|j\rangle
$$

for arbitrary phases $\phi_{j}$ and (dephasing) positive constants $r_{j}$ such that $\sum_{j} r_{j}=1$. Then

$$
\left\langle\chi_{s} \mid \chi_{t}\right\rangle=\sqrt{r_{s} r_{t}} \sum_{j} \omega_{j}^{t-s}=\sqrt{r_{s} r_{t}} n \delta_{s, t},
$$

which makes the vectors orthogonal. Further,

$$
\sum_{s}\left|\left(\chi_{s}\right)_{j}\right|^{2}=\sum_{s} r_{s}=1
$$

which makes the corresponding completely positive map trace preserving. The Kraus operators of this map are

$$
b_{s}=\sqrt{r_{s}} \sum_{j} e^{-i\left(\phi_{j}+2 \pi j s / n\right)}|j\rangle\langle j| .
$$


The map without dephasing (a pure rotation) is recovered by choosing $r_{s}=\delta_{s, 0}$.

All diagrams we have used can equally well be interpreted in (dagger compact closed) categories other than tensor networks of Hilbert spaces. In the context of category theory, the morphism corresponding to the entangling gate $\delta$ is called a classical structure, because it abstracts the process of copying classical information. It also abstracts a choice of orthonormal basis [82, 83]. The corresponding "equal superposition" state o- is the unique unit of the classical structure. Sequentialization and parallelization of commuting morphisms are consequences of the generalized spider theorem [84], which is deduced by similar diagrammatic manipulations. Further, a dagger compact closed category with completely positive morphisms can be constructed from any dagger compact closed category [85], giving the corresponding abstract "dephasing morphisms" that commute with the classical structure. See [86] for more information. Therefore our results have consequences in other theories. For example, conformal or topological quantum field theories can be formulated in terms of dagger compact closed categories of cobordisms 87 90. Similarly, geometric quantization can be formulated as concerning classical structures in the dagger compact category of symplectic manifolds and canonical relations [91]. Our results, giving classes of sequentializable configurations, become interesting in those settings when reading sequentializability as the ability to "trade entanglement for time".

In summary, we have shown that quantum maps composed of phase gates and general dephasing are exactly the maps that commute with the entangling map. This implies that the sequential and quantum protocols are equivalent. In particular, they are equally sensitive to noise and have exactly the same responsiveness for quantum metrology. The entangled (parallel) protocol is better suited for rapidly changing signals, but is technically challenging. The diagrammatical derivation we presented has advantages over direct computations: it is completely general and, though perhaps unfamiliar, arguably simpler. Also, the abstract point of view sheds some light on the fundamental structures of some quantum protocols and applies to other theories.

Acknowledgement. Much of this work has been done while both authors were at the Institute for Quantum Information at the California Institute of Technology. We thank Peter Selinger for pointing out 92], and David Pérez García, Robert König, Peter Love and Spiros Michalakis for discussions. SB acknowledges support from FIS2008-01236 and from Defense Advanced Research Projects Agency award N66001-09-1-2101. CH was supported by the Netherlands Organisation for Scientific Research (NWO).

\section{Appendix A: Bound on the Fisher information scaling for separable states}

The quantum Cramér-Rao inequality [5] states that

$$
\delta \hat{\phi} \geq \frac{1}{\sqrt{\mathcal{I}_{n}}}
$$

where $\mathcal{I}_{n}$ is the (quantum) Fisher information corresponding to the evolution of the quantum state parametrized by $\phi$. This appendix proves $\mathcal{I}_{n} \leq n \mathcal{I}_{\text {bound }}$ for separable states, where $\mathcal{I}_{\text {bound }}$ is a bound on the Fisher information for one system. This is a well known fact, which we include here for completeness (see e.g. 38] for a different proof). We also note that for subsystems evolving with Hamiltonian $h$ (so the total Hamiltonian is $\sum h$ ) there exists a bound $\mathcal{I}_{\text {bound }} \leq\|h\|[26,31$.

Consider a quantum state $\rho$, implicitly parametrized by $\phi$. The quantum Fisher information for the state $\rho$ is defined as

$$
\mathcal{I}_{n}=\operatorname{Tr} \rho \mathcal{L}_{n}^{2}
$$

where the symmetric logarithmic derivative $\mathcal{L}$ is the Hermitian operator implicitly defined by the equation

$$
\frac{1}{2}\left(\mathcal{L}_{n} \rho+\rho \mathcal{L}_{n}\right)=\frac{\partial \rho}{\partial \phi} .
$$

If follows directly from this definition that

$$
\operatorname{Tr} \rho \mathcal{L}=\operatorname{Tr} \frac{1}{2}\left(\mathcal{L}_{n} \rho+\rho \mathcal{L}_{n}\right)=\operatorname{Tr} \frac{\partial \rho}{\partial \phi}=\frac{\partial \operatorname{Tr} \rho}{\partial \phi}=0 .
$$

Now assume that $\rho$ is a product quantum state $\rho_{p}=$ $\rho^{(1)} \otimes \cdots \otimes \rho^{(n)}$. It is easy to check directly that if $\mathcal{L}^{(j)}$ is the symmetric logarithmic derivative corresponding to $\rho^{(j)}$, then the symmetric logarithmic derivative of the product state $\rho$ is

$$
\mathcal{L}_{p}=\sum_{j} 1 \otimes \cdots \otimes 1 \otimes \mathcal{L}^{(j)} \otimes \cdots \otimes 1=\sum_{j} \mathcal{L}^{(j)} .
$$

The corresponding Fisher information is

$$
\begin{aligned}
\mathcal{I}_{p} & =\operatorname{Tr} \rho_{p} \mathcal{L}_{p}^{2}=\sum_{j} \operatorname{Tr} \rho_{p}\left(\mathcal{L}^{(j)}\right)^{2}+\sum_{j \neq k} \operatorname{Tr} \rho_{p} \mathcal{L}^{(j)} \otimes \mathcal{L}^{(k)} \\
& =\sum_{j} \operatorname{Tr} \rho_{p}\left(\mathcal{L}^{(j)}\right)^{2} \\
& =\sum_{j} \mathcal{I}_{p}^{(j)} \leq n \mathcal{I}_{\text {bound }} .
\end{aligned}
$$

The fact that $\operatorname{Tr} \rho_{p} \mathcal{L}^{(j)} \otimes \mathcal{L}^{(k)}=0$ for $j \neq k$ follows almost directly from (A2).

Finally consider an ensemble of states $\rho_{e}=\sum_{j} p_{j} \rho_{j}$. To calculate the symmetric logarithmic derivative of an ensemble it is simpler to start with the corresponding quantum superposition $\rho_{e x}=\sum_{j} p_{j} \rho_{j} \otimes|j\rangle\langle j|$. It is easy to check directly that the corresponding symmetric logarithmic derivative for this superposition is 
$\mathcal{L}_{e x}=\sum_{j} \mathcal{L}^{(j)} \otimes|j\rangle\langle j|$. This gives the quantum Fisher information for the superposition

$$
\mathcal{I}_{e x}=\operatorname{Tr} \rho_{e x} \mathcal{L}_{e x}^{2}=\sum_{j} p_{j} \mathcal{I}_{e x}^{(j)} \leq \max _{j} \mathcal{I}_{e x}^{(j)}
$$

Now, the difference between the quantum ensemble $\rho_{e}$ and the quantum superposition $\rho_{e x}$ is simply a trace over the auxiliary system that marks the particular state of the ensemble. That is, we forget or lose information. The corresponding Fisher information can only decrease as a result: $\mathcal{I}_{e} \leq \mathcal{I}_{e x}$. Formally, this is a consequence of the monotonicity of the Fisher information [93].

To conclude, we recall that a separable quantum state has the form $\rho_{s}=\sum_{j} p_{j} \rho_{1}^{(j)} \otimes \cdots \otimes \rho_{N}^{(j)}$. Putting together the bounds for ensembles and product states we conclude that $\mathcal{I}_{s} \leq n \mathcal{I}_{\text {bound }}$, or, in other words,

$$
\delta \hat{\phi} \geq \frac{1}{\sqrt{n \mathcal{I}_{\text {bound }}}} .
$$

\section{Appendix B: Diagrammatic proofs}

Diagrammatically, entangling gates are axiomatically defined by the following properties, called associativity, isometry, commutativity, and Frobenius law [77, 84].

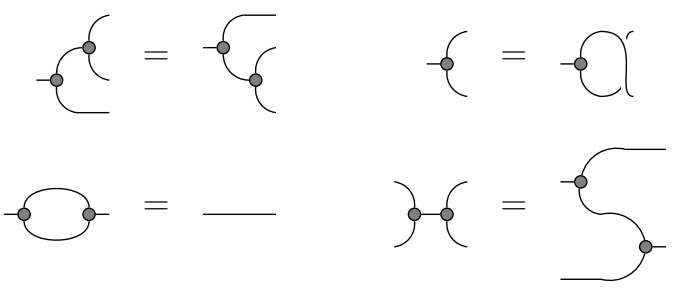

It then follows [83] from the structure of compact categories that there exists a unique morphism $\multimap$ satisfying

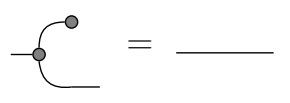

Now Eq. (3) follows immediately from associativity:

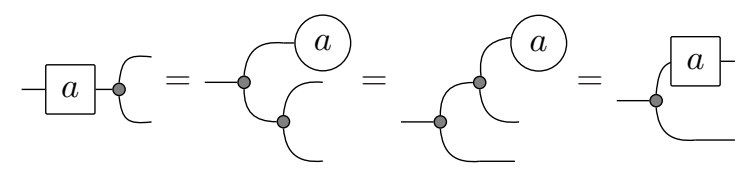

The transformation between the parallel and sequential protocols (5) and (6), as explicitly given in Eq. (7), follow directly from isometry:

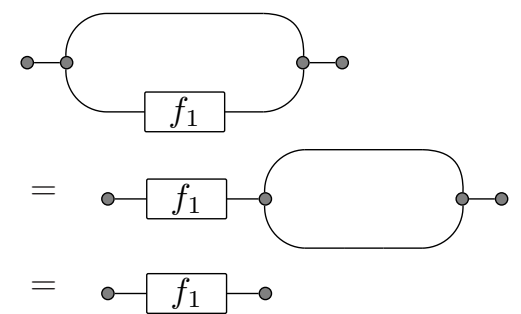

The bijection between the states and operators of Eqs. (8) and (2) is easily seen using the unit property (B1).

Finally, the diagrammatic proof of the commutativity of the linear operators or quantum maps of Eq. (8) can now easily be seen to follow from the associative and commutative properties of the given classical structure.

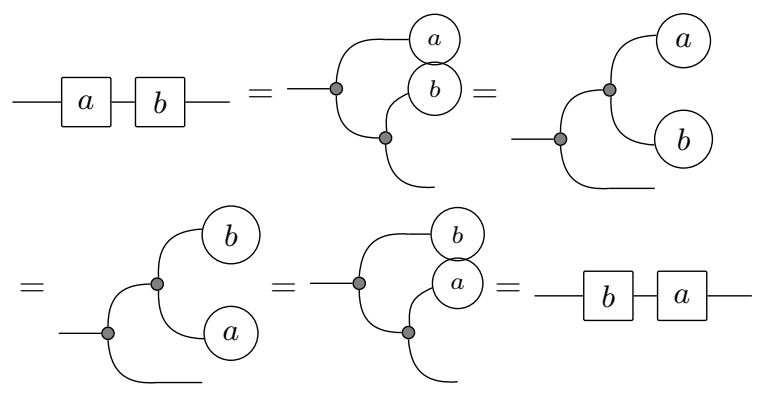

These proofs only rely on the four axioms of classical structures, and hence hold in any dagger compact category rather than just for finite-dimensional Hilbert spaces with either linear or quantum maps.

052106 (2001).

[1] C. W. Helstrom, Quantum detection and estimation theory (Academic Press, New York, 1976).

[2] C. M. Caves, Phys. Rev. D 23, 1693 (1981).

[3] A. S. Holevo, Probabilistic and statistical aspects of quantum theory (North-Holland, Amsterdam, 1982).

[4] D. J. Wineland et. al. , Phys. Rev. A 46, R6797 (1992).

[5] S. L. Braunstein and C. M. Caves, Phys. Rev. Lett. 72, 3439 (1994).

[6] J. J. Bollinger et. al. , Phys. Rev. A 54, 4649 (1996).

[7] S. F. Huelga et. al., Phys. Rev. Lett. 79, 3865 (1997).

[8] V. Buzek, R. Derka, and S. Massar, Phys. Rev. Lett. 82, 2207 (1999).

[9] V. Meyer et. al., Phys. Rev. Lett. 86, 5870 (2001).

[10] D. Ulam-Orgikh and M. Kitagawa, Phys. Rev. A 64,
[11] A. Fujiwara, Phys. Rev. A 65, 12316 (2001).

[12] H. Lee et. al., J. Mod. Opt. 49, 2325 (2002).

[13] T. Rudolph and L. Grover, Phys. Rev. Lett. 91, 217905 (2003).

[14] M. W. Mitchell, J. S. Lundeen, and A. M. Steinberg, Nature 429, 161 (2004).

[15] P. Walther et. al., Nature 429, 158 (2004).

[16] A. André, A. S. Sorensen, and M. D. Lukin, Phys. Rev. Lett. 92, 230801 (2004).

[17] A. Luis, Phys. Lett. A 329, 8 (2004).

[18] E. Bagan, M. Baig, and R. Munoz-Tapia, Phys. Rev. A 70, 030301 (2004).

[19] J. A. Dunningham and K. Burnett, Phys. Rev. A 70, 
033601 (2004).

[20] G. Chiribella et. al., Phys. Rev. Lett. 93, 180503 (2004).

[21] J. Beltran and A. Luis, Phys. Rev. A 72, 045801 (2005).

[22] M. de Burgh and S. D. Bartlett, Phys. Rev. A 72, 042301 (2005).

[23] S. Boixo et. al., Laser Phys. 16, 1525 (2006).

[24] A. Monras, Phys. Rev. A 73, 033821 (2006).

[25] M. Hayashi, Phys. Lett. A 354, 183 (2006).

[26] V. Giovannetti, S. Lloyd, and L. Maccone, Phys. Rev. Lett. 96, 010401 (2006).

[27] A. Fujiwara, J. Phys. A 39, 12489 (2006).

[28] M. Hayashi, Quantum Information: An Introduction (Springer, 2006).

[29] B. L. Higgins et. al., Nature 450, 393 (2007).

[30] A. Shaji and C. M. Caves, Phys. Rev. A 76, 032111 (2007).

[31] S. Boixo et. al., Phys. Rev. Lett. 98, 090401 (2007).

[32] A. M. Rey, L. Jiang, and M. D. Lukin, Phys. Rev. A 76, 053617 (2007).

[33] W. van Dam et. al. , Phys. Rev. Lett. 98, 090501 (2007).

[34] A. Monras and M. G. A. Paris, Phys. Rev. Lett. 98, 160401 (2007).

[35] T. Nagata et. al., Science 316, 726 (2007).

[36] S. Boixo et. al., Phys. Rev. Lett. 101, 040403 (2008).

[37] S. Choi and B. Sundaram, Phys. Rev. A 77, 053613 (2008).

[38] S. Boixo et. al. , Phys. Rev. A 77, 012317 (2008).

[39] A. Fujiwara and H. Imai, J. Phys. A 41, 255304 (2008).

[40] L. Pezz and A. Smerzi, Phys. Rev. Lett. 100, 073601 (2008).

[41] J. A. Jones et. al., Science 324, 1166 (2009).

[42] J. Appel et. al., Proceedings of the National Academy of Sciences 106, 10960 (2009).

[43] B. A. Chase et. al., Phys. Rev. A 79, 062107 (2009).

[44] D. Maldonado-Mundo and A. Luis, Phys. Rev. A 80, 063811 (2009).

[45] U. Dorner et. al., Phys. Rev. Lett. 102, 040403 (2009).

[46] T. Lee et. al., Phys. Rev. A 80, 063803 (2009).

[47] M. Aspachs et. al., Phys. Rev. A 79, 033834 (2009).

[48] L. Maccone and G. De Cillis, Phys. Rev. A 79, 023812 (2009).

[49] C. M. Caves and A. Shaji, Opt. Comm. 283, 695 (2010).

[50] M. G. Genoni, S. Olivares, and M. G. A. Paris, Phys. Rev. Lett. 106, 153603 (2011).

[51] M. Kacprowicz et. al., Nat Photon 4, 357 (2010).

[52] K. Modi et. al. , arXiv:1003.1174 (2010).

[53] T. Tilma et. al., Phys. Rev. A 81, 022108 (2010).

[54] A. Rivas and A. Luis, Phys. Rev. Lett. 105, 010403 (2010).

[55] M. Zwierz, C. A. Pérez-Delgado, and P. Kok, Phys. Rev. Lett. 105, 180402 (2010).

[56] J. Kolstrokodynacuteski and R. DemkowiczDobrzanacuteski, Phys. Rev. A 82, 053804 (2010).

[57] V. Giovannetti, S. Lloyd, and L. Maccone, Nat Photon 5, 222 (2011).

[58] B. M. Escher, R. L. de Matos Filho, and L. Davidovich, Nat Phys 7, 406 (2011).

[59] M. Napolitano et. al., Nature 471, 486 (2011).

[60] M. Mullan and E. Knill, 1107.5347 (2011).

[61] A. Y. Kitaev, quant-ph/9511026 (1995).

[62] E. Knill, G. Ortiz, and R. D. Somma, Phys. Rev. A 75, 012328 (2007).

[63] R. B. Griffiths and C. Niu, Phys. Rev. Lett. 76, 3228 (1996).
[64] C. Moore and M. Nilsson, SIAM J. Comp. 31, 799 (2002).

[65] P. Hoyer and R. Spalek (Springer-Verlag, 2003), STACS '03, p. 234246, ACM ID: 696323.

[66] N. Schuch et. al., Phys. Rev. Lett. 98, 140506 (2007).

[67] D. Perez-Garcia et. al., Qauntum Inf. Comput. 7, 401 (2007), quantum Inf. Comput. 7, 401 (2007).

[68] G. Vidal, Phys. Rev. Lett. 99, 220405 (2007).

[69] F. Verstraete, V. Murg, and J. I. Cirac, Adv. Phys. 57, 143 (2008).

[70] C. M. Dawson, J. Eisert, and T. J. Osborne, Phys. Rev. Lett. 100, 130501 (2008).

[71] J. I. Cirac and F. Verstraete, J.Physics A 42, 504004 (2009).

[72] G. Evenbly and G. Vidal, arXiv:1106.1082 (2011).

[73] S. Mukamel, Principles of Nonlinear Optical Spectroscopy (Oxford University Press, USA, 1999).

[74] G. Gutoski and J. Watrous, in Proceedings of the thirtyninth annual ACM symposium on Theory of computing (ACM, San Diego, California, USA, 2007), pp. 565-574.

[75] G. Chiribella, G. M. D'Ariano, and P. Perinotti, Phys. Rev. Lett. 101, 060401 (2008).

[76] G. Chiribella et. al., Phys. Rev. A 80, 022339 (2009).

[77] B. Coecke and É. O. Paquette, in New structures for Physics (Springer, 2010), vol. 813 of Lecture Notes in Physics.

[78] P. Selinger, in New Structures for Physics (Springer, 2010), vol. 813 of Lecture Notes in Physics.

[79] S. Abramsky and B. Coecke, in Logic in Computer Science 19 (IEEE Computer Society, 2004), pp. 415-425.

[80] A. Joyal and R. Street, Advances in Mathematics 88, 55 (1991).

[81] A. Joyal and R. Street, Advances in Mathematics 102, 20 (1993).

[82] B. Coecke, D. Pavlović, and J. Vicary, Mathematical Structures in Computer Science (2009).

[83] S. Abramsky and C. Heunen, Clifford Lectures, AMS Proceedings of Symposia in Applied Mathematics (2011).

[84] B. Coecke and R. Duncan, in Automata, Languages and Programming, ICALP 2008 (Springer, 2008), no. 5126 in Lecture Notes in Computer Science, pp. 298-310.

[85] P. Selinger, in Quantum Programming Languages (Elsevier, 2007), vol. 170 of Electronic Notes in Theoretical Computer Science, pp. 139-163.

[86] C. Heunen and S. Boixo, to appear in the EPTCS proceedings of QPL (2011).

[87] J. Kock, Frobenius algebras and 2-D Topological Quantum Field Theories, no. 59 in London Mathematical Society Student Texts (Cambridge University Press, 2003).

[88] J. C. Baez, Structural foundations of quantum gravity (Oxford University Press, 2006), chap. Quantum quandaries: a category-theoretic perspective, pp. 240-265.

[89] M. F. Atiyah, Publications Mathematiques de l'I.H.É.S. 68, 175 (1988).

[90] G. Segal, Topology, Geometry and Quantum Field Theory (Cambridge University Press, 2004), chap. The definition of conformal field theory, pp. 421-577, no. 308 in London Mathematical Society Lecture Note Series.

[91] N. P. Landsman, Contemporary Mathematics 315, 9 (2002).

[92] M. B. McCurdy and P. Selinger (2006), unpublished manuscript, Dalhousie University.

[93] D. Petz, Linear algebra and its applications 244, 81 (1996). 